Prahlad K. Sethi

Nitin K. Sethi

Josh Torgovnick

Edward Arsura

\section{Cysticercosis of temporalis muscle: an unusual cause of temporal headaches. A case report}

Received: 31 August 2007

Accepted in revised form: 14 September 2007

Published online: 25 October 2007

\section{N.K. Sethi (凶)}

Department of Neurology,

NYP-Weill Cornell Medical Center,

525 East 68th Street,

New York, NY 10021, USA

e-mail: sethinitinmd@hotmail.com

Tel.: +1-646-515-5168

\section{P.K. Sethi}

Department of Neurology,

Sir Ganga Ram Hospital,

New Delhi, India

J. Torgovnick

Department of Neurology,

Saint Vincent's Hospital and Medical

Centers,

New York, NY, USA

\section{E. Arsura}

Department of Medicine,

Saint Vincent's Hospital and Medical

Centers,

New York, NY, USA
Abstract Cysticercosis is a common parasitic infection caused by encysted larvae of the helminth Taenia solium (pork tapeworm). The central nervous system (CNS) is the most important primary site of infection and the disease can present with solitary or multiple space occupying lesions. Less common presentations in the CNS include the racemose variety with macroscopic groups of cysticerci in the subarachnoid space giving the appearance of a cluster of grapes and basal or ventricular cysticercosis causing obstructive hydrocephalus. Involvement of other organs: skeletal muscle, eyes, myocardium and the lungs has also been reported. Cases of cysticercosis presenting as isolated muscle mass (pseudotumours) without involvement of the CNS have also been recently described in the literature. We present a case of a 43-year-old woman who complained of subacute onset of left temporal pain and headache. Magnetic resonance imaging (MRI) showed characteristic imaging findings suggestive of cysticercosis of the temporalis muscle.
Keywords Temporal headaches • Cysticercosis - Giant cell arteritis • Temporal arteritis

\section{Case report}

A 43-year-old woman presented to the out-patient neurology department for evaluation of left temporal pain and headache of 3 weeks' duration. She reported feeling a lump in the left temporal area for the past week. There were no complaints of blurring of vision or jaw claudication and she denied any myalgias. Examination of the patient revealed tenderness around the left temple with visible swelling. Neurological examination was normal with no motor or sensory deficits. Fundoscopic examination revealed a normal optic disc. The 
patient was empirically started on corticosteroids for a working diagnosis of temporal arteritis, and out-patient blood tests and magnetic resonance imaging (MRI) brain were ordered. MRI revealed a bulky left temporalis muscle with a $1.1 \times 1.3 \mathrm{~cm}$ intramuscular ring enhancing cystic lesion (Figs. 1-3). Cysticercosis serology with enzyme-linked immunosorbent assay (ELISA) was positive and erythrocyte sedimentation rate (ESR) was 34. No evidence of disseminated or intracranial cysticercosis was found. She was treated with $15 \mathrm{mg} / \mathrm{kg} /$ day of albendazole for 28 days with complete resolution of symptoms.

\section{Discussion}

Cysticercosis is a zoonotic disease caused by the helminth $T$. solium. The disease is endemic in developing countries such as India in South-east Asia. Cysticercosis demonstrates a strong predilection for the central nervous system (CNS) and it is the most common cause of acquired epilepsy in India. Apart from the CNS, other organs such as skeletal muscle, the eyes, the heart and the lungs may be involved either on their own or as a part of disseminated disease. Solitary skeletal muscle or soft tissue cysticercosis has been reported and there is a report of isolated involvement of the temporalis muscle [1]. The authors reported a 16-year-old woman who presented with a left temporal swelling of 7-year duration. A computed tomography (CT) scan of the head showed a $1.5 \times 1.3 \times 0.85 \mathrm{~cm}$ size lesion. Excision of the left temporalis muscle was done which revealed cysticercosis [2]. Our patient presented with subacute onset of left temporal pain and headache of 3 weeks' duration. Even though she was not in the age group commonly afflicted by temporal arteritis, we empirically treated her with steroids while awaiting the results of ancillary investigations like ESR and brain MRI.

Routine laboratory testing is generally unremarkable in cysticercosis. The diagnosis rests on characteristic imaging findings supported by serologic tests such as enzyme-linked immunosorbent assay (ELISA) and enzyme-linked immunoelectrotransfer blot (EITB). The sensitivity of serum ELISA

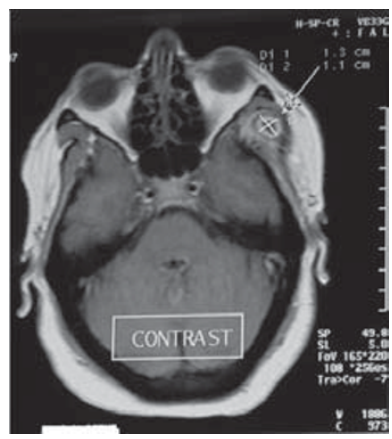

Fig. 1 Axial T1 weighted MRI with contrast showing a $1.1 \times 1.3 \mathrm{~cm}$ intramuscular enhancing lesion.

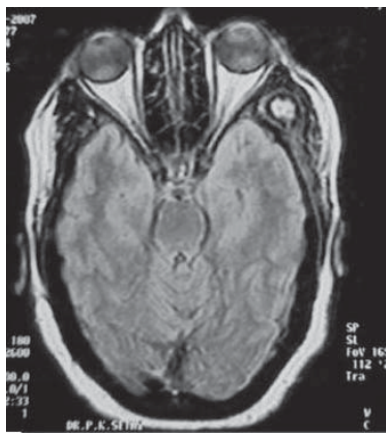

Fig. 2 Axial T1 weighted MRI with contrast showing a $1.1 \times 1.3 \mathrm{~cm}$ intramuscular enhancing lesion in the left temporalis muscle.

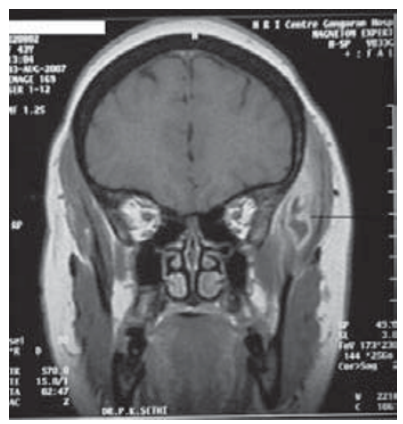

Fig. 3 Coronal T1 weighted enhanced MRI showing a bulky left temporalis muscle with an enhancing cystic lesion.

varies from $50 \%$ to $87 \%$ with a specificity of $63 \%-95 \%$. EITB, on the other hand, has a specificity approaching $100 \%$. For patients residing in endemic regions, the imaging characteristics on CT and MRI usually suffice in establishing a diagnosis of cysticercosis. Histological and serological confirmation is not required in this clinical setting [2].

\section{References}

1. Ergen FB, Turkbey B, Kerimoglu U et al (2005) Solitary cysticercosis in the intermuscular area of the thigh: a rare and unusual pseudotumor with characteristic imaging findings. J Comput Assist Tomogr 29:260-263
2. Kumar R, Singh V, Rastogi A (2005) Cysticercosis of temporalis muscle: a case report. J Ped Neurol 3:269-272 